\section{TATRA \\ MOUNTaiNS \\ Mathematical Publications}

DOI: $10.2478 / \mathrm{tmmp}-2013-0027$

Tatra Mt. Math. Publ. 56 (2013), 61-66

\title{
ON BUCK'S UNIFORM DISTRIBUTION IN COMPACT METRIC SPACES
}

\author{
MILAN PAŠTÉKA
}

\begin{abstract}
We define uniform distribution in compact metric space with respect to the Buck's measure density originated in [Buck, R. C.: The measure theoretic approach to density, Amer. J. Math. 68 (1946), 560-580]. Weyl's criterion is derived. This leads to an existence result.
\end{abstract}

The notion of uniformly distributed sequence is originated by Hermann Weyl in 1916 in the paper WEY for the real valued sequences. In this paper only the fractional parts of elements of given sequence are substantial. Thus the object of observations are the elements of the unit interval. Let us remark Weyl use the expression uniform distribution modulo 1. Later this notion was generalizes for compact metric and topological spaces. For the survey we refer to the monographs $[\mathrm{H}], \mathrm{K}-\mathrm{N}], \mathrm{D}-\mathrm{T}], \mathrm{P}]$.

Denote by $\mathbb{N}$ the set of positive integers. If for a subset $A \subset \mathbb{N}$ there exists the limit

$$
\lim _{n \rightarrow \infty} \frac{\operatorname{card}\{k \in A ; k \leq n\}}{n}:=d(A)
$$

then we say that the set $A$ has the asymptotic density and we call the value $d(A)$ the asymptotic density of $A$. This set function is finitely additive probability measure and its properties are described for instance in NAR, $\mathrm{P}$, PAS3].

Let $(\mathbb{M}, \rho, \lambda)$ be the compact metric space with the metric $\rho$ and Borel measure $\lambda$. Suppose that $\left\{x_{n}\right\}$ is a sequence and $S$ an arbitrary set. Denote

$$
A\left(S,\left\{x_{n}\right\}\right)=\left\{n \in \mathbb{N} ; x_{n} \in S\right\} .
$$

It is well known that the sequence $\left\{x_{n}\right\}$ of elements of $\mathbb{M}$ is called uniformly distributed if and only if for every measurable set $S \subset \mathbb{M}$ such that $\lambda(\partial S)=0$, there holds that $A\left(S,\left\{x_{n}\right\}\right)$ has asymptotic density equal $\lambda(S)$, (see [WEY], $\mathrm{K}-\mathrm{N}], \mathrm{D}-\mathrm{T}], \mathrm{P})$. A measurable set $S$ which fulfils the condition $\lambda(\partial S)=0$ is called the set of $\lambda$-continuity. The following is well-known characterization of uniform distribution.

(C) 2013 Mathematical Institute, Slovak Academy of Sciences. 2010 Mathematics Subject Classification: 11B05, 11K06.

Keywords: density, uniform distribution.

The research was supported by the grant VEGA 2/0206/10. 


\section{MILAN PAŠTÉKA}

WEYL'S CRITERION. Let $\left\{x_{n}\right\}$ be a sequence of elements of $\mathbb{M}$. This sequence is uniformly distributed if and only if

$$
\lim _{N \rightarrow \infty} \frac{1}{N} \sum_{n=1}^{N} f\left(x_{n}\right)=\int f d \lambda
$$

for every real valued continuous function $f$ defined on $\mathbb{M}$.

In this paper we shall study the special case of uniform distribution related to other type of finitely additive probability measure, so called Buck's measure density constructed in BUC, 1946]. Put $r+(m)=\{a \in \mathbb{N} ; a \equiv r(\bmod m)\}$, for $m \in \mathbb{N}, r$-integer. Let $S \subset \mathbb{N}$. The value

$$
\mu^{*}(S)=\inf \left\{\sum_{j=1}^{s} \frac{1}{m_{j}} ; S \subset r_{1}+\left(m_{1}\right) \cup \cdots \cup r_{s}+\left(m_{s}\right), s \in \mathbb{N}\right\}
$$

is called the measure density of the set $S$. The system of sets

$$
\mathcal{D}_{\mu}=\left\{S \subset \mathbb{N} ; \mu^{*}(S)+\mu^{*}(\mathbb{N} \backslash S)=1\right\}
$$

is the set algebra and the restriction $\mu=\left.\mu^{*}\right|_{\mathcal{D}_{\mu}}$ is the finitely additive probability measure on $\mathcal{D}_{\mu}$.

A sequence $\left\{x_{n}\right\}$ of the elements of $\mathbb{M}$ is called Buck's uniformly distributed if for every set of $\lambda$-continuity $S$ we have

$$
A\left(S,\left\{x_{n}\right\}\right) \in \mathcal{D}_{\mu} \quad \text { and } \quad \mu\left(A\left(S,\left\{x_{n}\right\}\right)\right)=\lambda(S) .
$$

We say that a sequence of positive integers $\left\{k_{n}\right\}$ is uniformly distributed if for every $m \in \mathbb{N}$ and integer $r A\left(r+(m),\left\{k_{n}\right\}\right)$ has asymptotic density equal $\frac{1}{m}$. In [PAS3, Theorem 7, p. 49], the following characterization of the algebra $\mathcal{D}_{\mu}$ is given.

Theorem A. Arbitrary set $H \subset \mathbb{N}$ belongs to $\mathcal{D}_{\mu}$ if and only if $A\left(H,\left\{k_{n}\right\}\right)$ has asymptotic density equal to $\mu^{*}(H)$ for every sequence of positive integers $\left\{k_{n}\right\}$ which is uniformly distributed.

Theorem A says that a set $H \subset \mathbb{N}$ belongs to $\mathcal{D}_{\mu}$ if and only if

$$
\lim _{N \rightarrow \infty} \sum_{n=1}^{N} \chi_{H}\left(k_{n}\right)=\mu^{*}(H)
$$

for every uniformly distributed sequence of positive integers $\left\{k_{n}\right\}$. Clearly for the sequence $\left\{x_{n}\right\}$ of elements of $\mathbb{M}$ and $A:=A\left(S,\left\{x_{n}\right\}\right)$ we have $\chi_{S}\left(X_{k}\right)=\chi_{A}(k)$. Thus the sequence $\left\{x_{n}\right\}$ is Buck's uniformly distributed if for every set of $\lambda$ -continuity $S$ there holds

$$
\lim _{N \rightarrow \infty} \frac{1}{N} \sum_{n=1}^{N} \chi_{S}\left(x_{k_{n}}\right)=\lambda(S),
$$




\section{ON BUCK'S UNIFORM DISTRIBUTION IN COMPACT METRIC SPACES}

and so by the same procedure as in the proof of Theorem in $[\mathrm{K}-\mathrm{N}]$ the following form of Weyl's criterion can be proved.

TheOREM 1. Let $\left\{x_{n}\right\}$ be a sequence of elements of $\mathbb{M}$. Then this sequence is Buck's uniformly distributed if and only if

$$
\lim _{N \rightarrow \infty} \frac{1}{N} \sum_{n=1}^{N} f\left(x_{k_{n}}\right)=\int f d \lambda
$$

for every real valued continuous function $f$ defined on $\mathbb{M}$ and for every sequence of positive integers $\left\{k_{n}\right\}$ which is uniformly distributed.

The implication $\Rightarrow$ follows from the fact that every continuous real valued function on $\mathbb{M}$ can be uniformly approximated by the functions in the form $c_{1} \chi_{S_{1}}+\cdots+c_{r} \chi_{S_{r}}$ where $S_{1}, \ldots, S_{r}$ are the sets of $\lambda$ continuity. The opposite implication can be proved by the Urysohn's lemma. For the details we refer to $\mathrm{K}-\mathrm{N}$.

Theorem 1 can be formulated in the equivalent form.

Let $\left\{x_{n}\right\}$ be a sequence of elements of $\mathbb{M}$. Then this sequence is Buck's uniformly distributed if and only if for every sequence of positive integers $\left\{k_{n}\right\}$ which is uniformly distributed the sequence $\left\{x_{k_{n}}\right\}$ is uniformly distributed, too.

A sequence $\left\{x_{n}\right\}$ of elements of $\mathbb{M}$ is called polyadically continuous if and only if for every $\varepsilon>0$ there exists $m \in \mathbb{N}$ that

$$
n_{1} \equiv n_{2} \quad(\bmod m) \Rightarrow \rho\left(x_{n_{1}}, x_{n_{2}}\right)<\varepsilon .
$$

Since every continuous real valued function on $\mathbb{M}$ is uniformly continuous we obtain that for a continuous function $f$ and polyadically continuous sequence $\left\{x_{n}\right\}$ of elements of $\mathbb{M}$ is the arithmetic function $g(n)=f\left(x_{n}\right)$ polyadically continuous, we obtain directly from the properties of polyadically continuous arithmetic functions, (see $[\mathrm{P}]$ ).

Corollary. A polyadically continuous sequence $\left\{x_{n}\right\}$ of elements of $\mathbb{M}$ is Buck's uniformly distributed if and only if it is uniformly distributed.

The existence of various type of uniformly distributed sequence in real case is provided by the construction of numerous examples. In more abstract cases the existence of such sequences should be provided by some arguments. In the paper [H] there is constructed uniformly distributed sequence in compact metric space. Motivated by this paper, using the same procedure we prove the following property.

TheOREM 2. There exists Buck's uniformly distributed sequence of the elements of $\mathbb{M}$. 


\section{MILAN PAŠTÉKA}

Proof. The compactness of $\mathbb{M}$ provides that there exists a system

$$
\mathcal{S}_{m}=\left\{S_{m}^{j}, j=1, \ldots, r(m)\right\}, \quad m=1,2 \ldots, \quad S_{m}^{j} \neq \emptyset
$$

of finite closed covers of $\mathbb{M}$, having the following properties:

i) If $n\left(\mathcal{S}_{m}\right)=\max \left\{\operatorname{diam}\left(S_{m}^{j}\right), j=1, \ldots, r(m)\right\}, m=1,2, \ldots$, then

$$
\lim _{m \rightarrow \infty} n\left(\mathcal{S}_{m}\right)=0 .
$$

ii) For every $m$ and $i \neq j$ there holds $\lambda\left(S_{m}^{i} \cap S_{m}^{j}\right)=0$.

iii) If $m \leq n$, then for every $i \leq r(n)$ there exists uniquely determined

$$
j=j(i) \leq r(m) \text { such that } S_{n}^{i} \subset S_{m}^{j} .
$$

iv) The indices in iii) are ordered in such a way that

$$
i_{1}<i_{2} \Rightarrow j\left(i_{1}\right) \leq j\left(i_{2}\right) .
$$

The property i) provides that every real valued continuous function defined on $\mathbb{M}$ can be uniformly approximated by the linear combination of the functions $\chi_{S_{m}^{j}}$, $j=1, \ldots, r(m)$ for suitable $m$.

The Van der Corput sequence defined by the power expansion of positive integers (see [K-N], PAS3]) is an example of the sequence of elements of interval $[0,1]$ which is Buck's uniformly distributed. Thus we can suppose that such sequence exists in this interval. Let $\left\{\gamma_{n}\right\}$ be a sequence Buck's uniformly distributed in $[0,1]$.

To each cover $\mathcal{S}_{m}$ we can associate the finite system of interval $I_{m}^{j}, j=1, \ldots$ $\ldots, r(m)$ such that

$$
I_{m}^{j}=\left[\sum_{l=1}^{j-1} \lambda\left(S_{m}^{l}\right), \sum_{l=1}^{j} \lambda\left(S_{m}^{l}\right)\right],
$$

thus $\left|I_{m}^{j}\right|=\lambda\left(S_{m}^{l}\right)$ and this system is division of the interval $[0,1]$ and we can suppose that the endpoints of $I_{m}^{k}, k=1, \ldots, r(m)$ do not coincide with the elements of $\left\{\gamma_{n}\right\}$. Each number $\alpha \in[0,1]$ which does not coincide with the endpoints of the intervals $I_{m}^{k}, k=1, \ldots, r(m)$ belongs to exactly one interval $I_{m}^{l(m)}$ for every $m=1,2, \ldots$, where $l(m):=l(m, \alpha)$ depends on $\alpha$. Since for every $m=1,2, \ldots$ we have

$$
S_{m+1}^{l(m+1)} \subset S_{m}^{l(m)},
$$

the compactness of $\mathbb{M}$ guaranties that the set $D(\alpha)=\cap_{m=1}^{\infty} S_{m}^{l(m)}$ is non empty. Consider now such sequence $\left\{x_{n}\right\}$ of elements of $\mathbb{M}$ that $x_{n} \in D\left(\gamma_{n}\right), n=$ $1,2, \ldots$ We prove that this sequence is Buck's uniformly distributed. Let $\left\{k_{n}\right\}$ be arbitrary uniformly distributed sequence of positive integers. Denote for $H \subset \mathbb{M}$ 


\section{ON BUCK'S UNIFORM DISTRIBUTION IN COMPACT METRIC SPACES}

$$
\begin{aligned}
& \lambda^{*}(H)=\limsup _{N \rightarrow \infty} \sum_{n=1}^{N} \chi_{H}\left(x_{k_{n}}\right), \\
& \lambda_{*}(H)=\liminf _{N \rightarrow \infty} \sum_{n=1}^{N} \chi_{H}\left(x_{k_{n}}\right) .
\end{aligned}
$$

Let $\mathcal{Z}$ denote the system of all sets of the form $\cup_{t=1}^{T} S_{m}^{i_{t}}$. Clearly

$$
\chi_{\cup_{t=1}^{T} S_{m}^{i_{t}}} \geq \chi_{\cup_{t=1}^{T} I_{m}^{i_{t}}}
$$

Thus using the fact that the sequence $\left\{x_{k_{n}}\right\}$ is uniformly distributed in $[0,1]$ we obtain that for every $S \in \mathcal{Z}$ we have $\lambda_{*}(S) \geq \lambda(S)$.

From the other side we consider that for every open set $G$ there holds

$$
\lambda(G)=\sup \{\lambda(S) ; S \subset G, S \in \mathcal{Z}\} .
$$

Thus for every closed set $F$ and every $\varepsilon>0$ there exists a set $S^{\prime} \in \mathcal{Z}$ that $S^{\prime} \subset \mathbb{M} \backslash F$ and $\lambda\left(S^{\prime}\right)>\lambda(\mathbb{M} \backslash F)-\varepsilon$. This yields

$$
\lambda^{*}(F) \leq 1-\lambda_{*}\left(S^{\prime}\right) \leq 1-\lambda\left(S^{\prime}\right)<1-\lambda(\mathbb{M} \backslash F)+\varepsilon=\lambda(F)+\varepsilon .
$$

Since $\varepsilon$ is arbitrary we obtain $\lambda^{*}(F) \leq \lambda(F)$. Therefore for every $S \in \mathcal{Z}$ it holds $\lambda_{*}(S)=\lambda^{*}(S)=\lambda(S)$ and the assertion follows from $\left.\mathrm{v}\right)$.

We conclude with following remark. Let $B$ be the set of all Buck's uniformly distributed sequences of elements of $\mathbb{M}$. Since each such sequence is well distributed (see [K-N], [N]) we obtain directly from the result of [N], (see also [K-N]), that $\lambda^{\infty}(B)=0$, where $\lambda^{\infty}$ is the product measure on the space of all sequences of elements of $\mathbb{M}$.

\section{REFERENCES}

[BUC] BUCK, R. C.: The measure theoretic approach to density, Amer. J. Math. 68 (1946), 560-580.

[D-T] DRMOTA, M.-TICHY, R. F.: Sequences, Discrepancies and Applications. Springer, Berlin, 1997.

[H] HEDRLIN, Z.: On integration in compact metric spaces, Comment. Math. Univ. Carolin. 2 (1961), 17-19.

[K-N] KUIPERS, L.-NIEDERREITER, H.: Uniform Distribution of Sequences, in: Pure Appl. Math. (N. Y.), John Wiley and Sons, New York, 1974.

[N] NIEDERREITER, H.: On the existence of uniformly distributed sequences in compact spaces, Compos. Math. 25 (1972), 93-99.

[NAR] NARKIEWICZ, W.: Teoria Liczb (Number Theory), in: Bibl. Mat., Vol. 50, PWN, Warszawa, 1977.

[P] POSTNIKOV, R. G.: Introduction to Analytic Number Theory, in: Transl. Math. Monogr., Vol. 68, Nauka, Moscov, 1971 (In Russian). English translation: Amer. Math. Soc., Providence, RI, 1981. 


\title{
MILAN PAŠTÉKA
}

[PAS1] PAŠTÉKA, M.: Remarks on one type of uniform distribution, Unif. Distrib. Theory 2 (2007), 79-92.

[PAS2] PAŠTÉKA, M.: Remarks on Buck's measure density, Tatra Mt. Math. Publ. 3 (1993), 191-200.

[PAS3] PAŠTÉKA, M.: On four approaches to density. to appear, 95 pages.

[WEY] WEYL, H.: Über die Gleichverteilung von Zahlen mod. Eins, Math. Ann. 77 (1916), $313-352$.

Received July 1, 2013

\author{
Department of Mathematics and \\ Computer Science \\ Faculty of Education \\ University of Trnava \\ Priemyselná 4 \\ SK-918-43 Trnava \\ SLOVAKIA \\ E-mail: pasteka@mat.savba.sk
}

